\title{
Digitalisierung im Werkzeug- und Formenbau als neue Wunderwaffe bei der Herstellung von Produkten!/?/!
}

\author{
Thomas Seul ${ }^{1(\bowtie)}$ und Fabian Diehr ${ }^{2}$ \\ ${ }^{1}$ Hochschule Schmalkalden, Schmalkalden, Deutschland \\ t. seulahs-sm.de \\ 2 wortundform GmbH, München, Deutschland
}

Schlüsselwörter: Werkzeug- und Formenbau · Produktionsprozesse · Digitalisierung

Prozesse verlieren zunehmend ihre Grenzschärfe. Sie überlagern und verzahnen sich von der Produktidee bis zur Serienfertigung ist die Expertise des Werkzeugmachers gefragt. Digitalisierung und Produktion sind dabei die beherrschenden Themen. Doch gilt es hier, die vielen Worthülsen greifbar zu machen. Gerade für die kleinen Betriebe in Deutschland ist es wichtig, ohne Vorbehalte die ersten Schritte tun zu können. Denn die Umbrüche in unserer Branche machen nicht vor dem 10-Mann-Betrieb halt, auch wenn es diesen bereits in dritter Generation gibt ... Wir müssen uns vielmehr der Effizienzforderung und der damit einhergehenden Vernetzung der Fertigungstechnik und der Logistik stellen! Wesentlich dabei ist, stets den Bezug zur Praxis aufzuzeigen. Die Werkzeugmacher-Branche in Deutschland lebt nämlich von den vielen kleinen „Hidden Champions“, die „Hands on“-Innovationen schaffen. Doch gerade hier kann Industrie 4.0 und Digitalisierung von Betrieb zu Betrieb ganz anders aussehen. Die einen beginnen mit Insellösungen, um diese sukzessive ,aufzubohren“, die anderen streben nach der durchgängigen Komplettlösung. Allesamt vereint sie, dass man sich den Herausforderungen der „,vierten industriellen Revolution“ stellen möchte, um weiterhin Spitzenreiter bei Qualität, Performance und Präzision zu sein.

Fakt ist aber auch, dass sich der Werkzeug- und Formenbau bereits seit Jahrzehnten mit 4.0 beschäftigt - nur wurde es bisher nicht so benannt. Die Digitalisierung zieht sich schon lange auf mehreren Ebenen durch alle Bereiche der Branche. Mehr noch: Die Unternehmen unseres Metiers waren die ersten, die die Digitalisierung ihrer Prozesse vorangetrieben haben: Vor 40 Jahren kamen im Werkzeug- und Formenbau die ersten CAD-/CAM-Systeme zum Einsatz. Die Elektronik veränderte in der Folge schnell die Organisation der Arbeit: Aus einer Fertigung, bei der der einzelne Werkzeugmacher alle Schritte betreute und als Generalist für das gesamte Werkzeug verantwortlich war, entwickelte sich eine arbeitsteilige Struktur, bei der sich der Einzelne auf sein Spezialisierungsfeld in der Herstellung des Werkzeugs fokussiert. Die Durchlaufzeiten von damals haben sich dadurch jedoch nicht zwangsläufig verkürzt, weil die Werkzeuge inzwischen wesentlich komplexer geworden sind. Hinzu kommt eine wesentlich erhöhte Präzision. Denn moderne Werkzeuge sind mittlerweile häufig wie skalierte Uhrwerke. 


\section{Ressourcen und Fähigkeiten durch effiziente Fertigungsabläufe von Werkzeugen sichern - Automatisierung in der Nische als Notwendigkeit}

Die Programme der Bundesregierung zum Thema Digitalisierung und Industrie 4.0 sind wichtig, richtig und auch notwendig. Das, was den Werkzeug- und Formenbau in Deutschland ausmachen muss, um in Zukunft überhaupt noch fertigungsfähig und wirtschaftlich aktiv zu sein, sind eine effiziente Fertigung und flexible Fertigungsprozesse. Diese Themen zeichnen den einzig richtigen Weg für unsere Branche.

Eine große Frage im Werkzeug- und Formenbau ist hierbei die nach der Automatisierung, die wir schon allein deswegen stellen müssen, weil immer weniger Menschen - Stichwort Fachkräftemangel - die hochpräzisen Fertigungstechnologien im Werkzeug- und Formenbau beherrschen. Und wer in Hochlohnländern die Chance hat, mit einer Schicht zwei oder gar drei Schichten im Span oder per Erosion zu befeuern, der sollte im Sinne von Effizienz und Wettbewerbsfähigkeit auf strukturiert organisierte Abläufe sowie die selektive Automatisierung von Prozessen setzen. Um als Betrieb die geeigneten Bereiche für die Automatisierung zu identifizieren, lohnt meist der Blick in die nähere Produktionshistorie. Jedes Unternehmen hat zwar seine ganz eigenen Spezialisierungen, aber eines ist bei fast allen gleich: die allzu menschlichen Problemzonen im Produktionsablauf. Genau hier kann die Automatisierung und die damit verbundene Digitalisierung des Shopfloors ansetzen und Fehler eliminieren. Wer nun auch noch die Herausforderung meistert, die dafür passenden Facharbeiter für die Planungsschicht anzuheuern, der schafft sich über die Standardisierung eine Entlastung bei Routinetätigkeiten und somit einen neuen Freiraum für Kreativität.

Automatisierung beeinflusst aber generell auch die Qualität im Sinne von Präzision und Wiederholbarkeit von Präzision - bei Oberflächeneigenschaften und bei der Maßhaltigkeit. Schwierig abzuwägen ist, an welchen Stellen man bewusst die Fertigungstoleranzen weiter öffnen kann, um wirtschaftlich - auch im Sinne des Kunden - zu agieren. Werkzeugmacher sollten sich daher strategisch überlegen, welche Toleranzen von der Produktseite in Zukunft von ihnen erwartet werden und welche Strukturen bzw. Technologien dazu passen. Eines muss dabei aber immer bedacht werden: Nicht IT oder Software wird in Zukunft unsere Produkte herstellen das macht immer noch das Werkzeug aus Stahl, das Menschen mit Maschinen in der erforderlichen Qualität und Spezifikation bearbeitet haben. Und diesen Werkzeugund Formenbauprozess gilt es zu beherrschen und zu validieren. Doch hierfür muss 
man erst die Hausaufgabe an der Basis machen. Ist der Prozess an sich marode, hilft Digitalisierung nichts - man erhält lediglich einen digitalisierten und immer noch maroden Prozess!

Werden die Bauteile, die aus den Werkzeugen und Formen herauskommen, geometrisch immer exakter, mit kleiner werdenden Toleranzen, bedingt das robuste Prozesse: Die Produktqualität muss wie an einer Perlenschnur aufgefädelt gewährleistet sein. Und das erreicht man wiederum nur mit der Präzision aus der digitalen Fertigung im Werkzeug- und Formenbau. Doch Qualitätsmerkmale beziehen sich nicht ausschließlich auf die Maßhaltigkeit. Es geht auch um die darauf aufbauende Prozessfähigkeit und Wiederholgenauigkeit in der Produktion für Bauteile, die mit diesen Werkzeugen hergestellt werden.

Hightech-Werkzeugbauunternehmen unterliegen daher heute durch die systematische, prozessorientierte und präventive Qualitätssicherung einer strengen Kontrolle. Die laufende Überprüfung der Ist- und Sollwerte ermöglicht automatisierte, durchgehende Prozesse für die Weiterverarbeitung der Werkstücke. Doch es stellt sich oft die Frage, wie präzise Werkzeugmacher noch werden müssen? Man hätte heute sicherlich nicht den Grad der Automatisierung im Spritzgießen oder beim Stanzen bzw. Umformen von Blechen und bei nachfolgenden Montageprozessen erreicht, wenn es die Präzision im Werkzeug nicht gäbe. Sie bestimmt, wie hoch der Output von komplexen Fertigungsabläufen ist, in Bezug auf die Qualität, Reproduzierbarkeit, Stabilität und Geschwindigkeit. Diesen Bereichen gilt es daher besondere Aufmerksamkeit zukommen zu lassen und die zugehörigen Prozesse im Detail zu dokumentieren, um sie auch nachweisen zu können.

Je genauer man bestimmte Maße und Toleranzen aber bestimmen kann, desto mehr wird das Haar in der Suppe gesucht. Wenn von Kundenseite Automatisierung und Industrie 4.0 in der Fertigung gefordert wird, dürfen nicht gleichzeitig Erstmusterprüfberichte mit hunderten taktil oder optisch geprüften Maßen eingefordert werden. Das ist eine technische veraltete Herangehensweise und spiegelt an vielen Stellen falsche Tatsachen wider. Vielmehr ist in erster Linie bei der Begutachtung der geometrischen Bauteilgüte der objektive Überblick über den Gesamtzusammenhang notwendig, um dann gegebenenfalls fokussiert die Details zu betrachten und zu diskutieren. Das große Ganze erfassen, dann Rückschlüsse daraus ziehen und nicht mit den granulierten Details beginnen ist effektiv und mit Sicherheit der effizientere Weg. Zeit und Wirtschaftlichkeit sind diesbezüglich die stichhaltigen Argumente und damit Wettbewerbsfaktoren. Mit der Etablierung digitaler Messtechnik, z. B. der Computertomographie (CT), steht ein Mess- und Analysewerkzeug zur Verfügung, um Produkt und Prozess zu optimieren. Die digitale Erfassung der Geometrie- und Bauteilstrukturen ermöglicht so die nachhaltige Digitalisierung des Produktentwicklungsund anschließenden Verifikationsprozesses. Mit CT erfasste, digitale Messdaten 
können im Entwicklungsprozess weitergegeben und genutzt werden. Erste Ansätze zu einer auf Künstlicher Intelligenz basierenden Verwendung der ermittelten Daten werden bereits in kooperativen Forschungsprojekten skizziert. Damit kann ein Beitrag zur Zukunftsfähigkeit von Wirtschaft und Forschung durch Stärkung der digitalen Produktentwicklung und Qualitätsüberwachung geschaffen werden (Abb. 1).
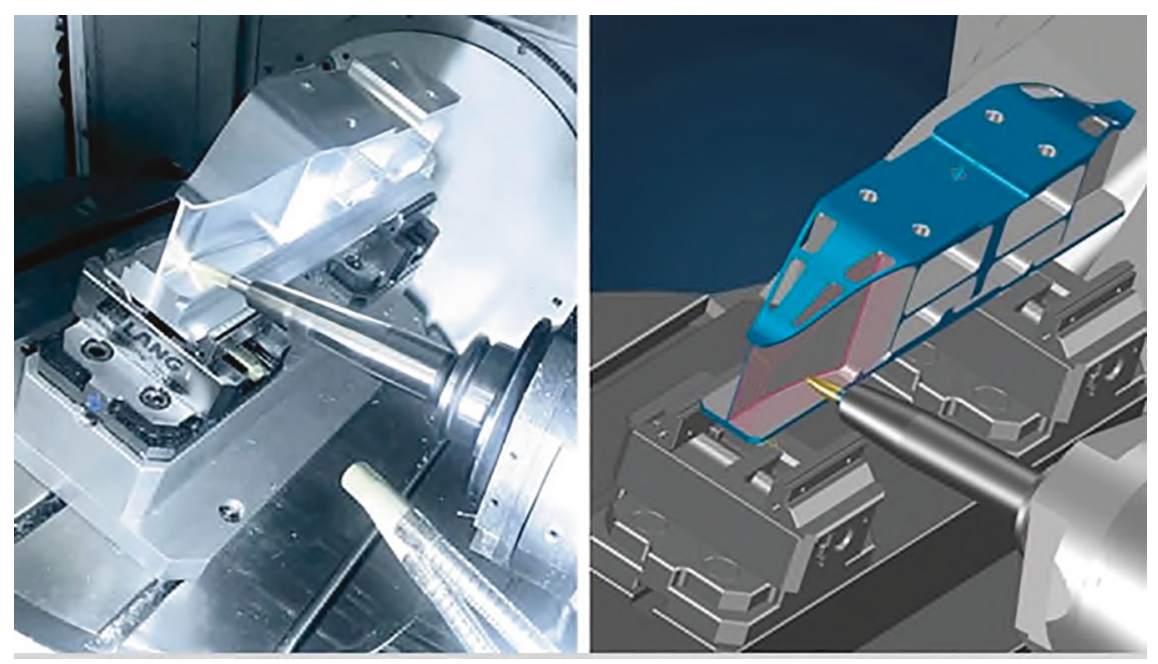

Abb. 1. Digitaler Zwilling. (Bild: Tebis AG, München)

Um Nutzen aus der Digitalisierung zu ziehen, muss man die neuen Anwendungen akzeptieren. Für den Werkzeug- und Formenbau war das Thema „digital“, ausgehend von der technischen Zeichnung bis hin zur Maschinensteuerung, immer eine Evolution, nie eine Revolution. Und weitere Entwicklungen werden auch eher mit der Verbesserung der Fertigungsprozesse und des Engineerings befassen. Ein wichtiges Arbeitsfeld im Bereich der Digitalisierung sind hierbei jedoch die Schnittstellen, die die vielen Satellitenlösungen am Markt verbindet. Das gesteckte Ziel lautet dabei: die umfassende Industrialisierung des Werkzeug- und Formenbaus. Dieser sollte dabei eine klare Vision und Strategie haben, eigenverantwortliche Mitarbeiter beschäftigen und über den gesamten Wertschöpfungsprozess Daten zur kontinuierlichen Verbesserung erfassen. Die Verwendung dieser Daten und das Management von Wissen und Erfahrungswerten steigert die Performance von 4.0-Unternehmen: Sie arbeiten effizient, realisieren kurze Durchlaufzeiten mit einer hohen Flexibilität und maximieren die Produktivität ihrer wertvollen Hightech-Betriebsmittel (Abb. 2). 


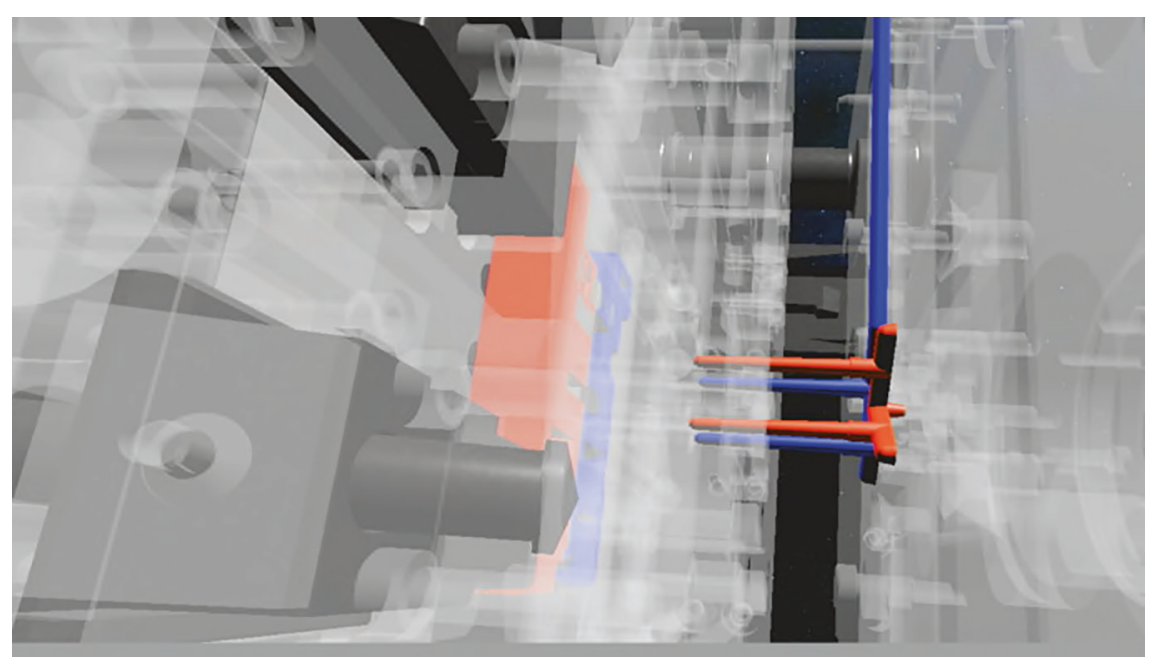

Abb. 2. Polyman-VR, eine Lernanwendung für Messen und Firmenevents. Der Polyman ist ein handliches Stück Kunststoff, das in der Ausbildung der Werkzeugmacher Anwendung findet: eine Zwei-Komponenten-Anwendung, die je zur Hälfte einer kunststoffgerechten und einer nicht kunststoffgerechten Bauteilkonstruktion entstammt. Alles Gute und alles Schlechte aus der Praxis der Spritzgussproduktion in einem Teil vereint und auf einen Blick erkennbar gemacht. Als vertiefende Anwendung ist das Polyman-Projekt auch per Virtual Reality zu bestaunen. Per 3D-Brille gelangt man in die virtuelle Realität des Lehrbauteils: Das gläsern animierte Werkzeug erlaubt es dem Betrachter, in den Spritzgussprozess und in die Kavität einzutauchen und hautnah die Dos and Don'ts der bauteilgerechten und kunststoffgerechten Werkzeugkonstruktion zu erleben. (Bild: wortundform $\mathrm{GmbH}$, München)

\section{Wettbewerbsvorteile durch intelligente Werkzeuge, eingebettet in das industrielle Umfeld}

Werkzeugmacher leben die Aspekte des 4.0 aber nicht nur in der Automation, sondern auch in vor- und nachgelagerten Prozessen - bei Partnern und bei Kunden. Das ist übrigens die Voraussetzung, um erfolgreich zu sein. Wesentlich bei der Betrachtung und Planung einer modernen Fertigung ist, dass Digitalisierungsstrategien dem Produktionsprozess folgen und nicht umgekehrt.

Bei der Digitalisierung in der industriellen Fertigung von Produkten kann die Werkzeug- und Formenbau-Branche dann beispielsweise mit intelligenten MonitoringSystemen die Schrittmacherrolle einnehmen. Wenn eingebettete Diagnosesysteme (EDS) prozessrelevante Daten erfassen und auswerten, können Werkzeuge dem Anwender aufzeigen, ob sein Fertigungsprozess im optimalen Betriebsfenster läuft oder davon abweicht. So kann nicht nur die Abmusterung beschleunigt werden, sondern der Kunde, also der 
Teilefertiger, erhält zusammen mit dem Werkzeug ein zuverlässiges Assistenzsystem zur Stabilisierung seiner Prozesse. Derartige „Powermoulds“ konfigurieren sich über eine Schnittstelle zur Spritzgießmaschine bzw. zur Presse idealerweise auch selbst und gleichen Referenzgrößen mit den tatsächlichen Prozessdaten in Echtzeit ab (Abb. 3 und 4).

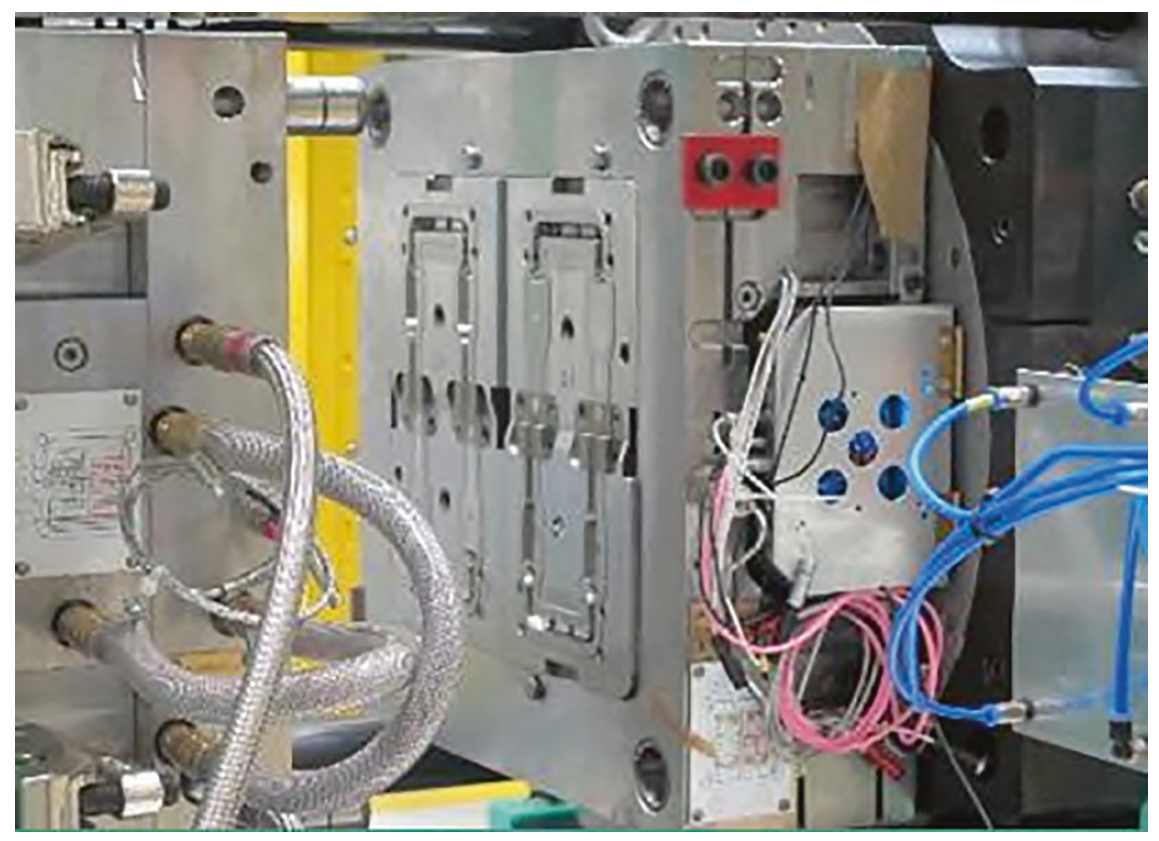

Abb. 3. Projekt Powermould an der Hochschule Schmalkalden. Mit einem fest installierten eingebetteten Diagnosesystem (EDS), inklusive integrierter Signalaufbereitung, wurden durch Methoden des maschinellen Lernens gezielte Entscheidungshilfen für den Maschinenbediener im Fehlerfall abgeleitet. In einem statistischen Versuchsablauf entwickelt das System über einen entsprechenden Algorithmus ein optimales Klassifikationsmodell, mit dem ,gute" und „schlechte“ Bauteile unterschieden werden können. Diejenigen Prozessparameter, die zu optimalen Bauteil-Merkmalen führen, werden als Referenzgrößen festgelegt und während der Produktion mit den tatsächlichen Prozessdaten in Echtzeit abgeglichen. Zusätzlich werden mit 3D-Scanner und anderen Geräten Qualitätseigenschaften der gefertigten Kunststoffprodukte untersucht und für die „Belehrung“ der computergestützten Auswertealgorithmen eingesetzt. Die 14 Sensoren ermitteln neben Produktionsdaten wie Werkzeuginnendruck, HeißkanalTemperatur, Temperiermittel-Temperatur oder Viskosität der Schmelze aber z. B. auch die Temperatur und die Luftfeuchtigkeit während der Lagerung. Alle Werte werden im akkubetriebenen EDS gespeichert. (Bild: Angewandte Kunststofftechnik, Hochschule Schmalkalden) 


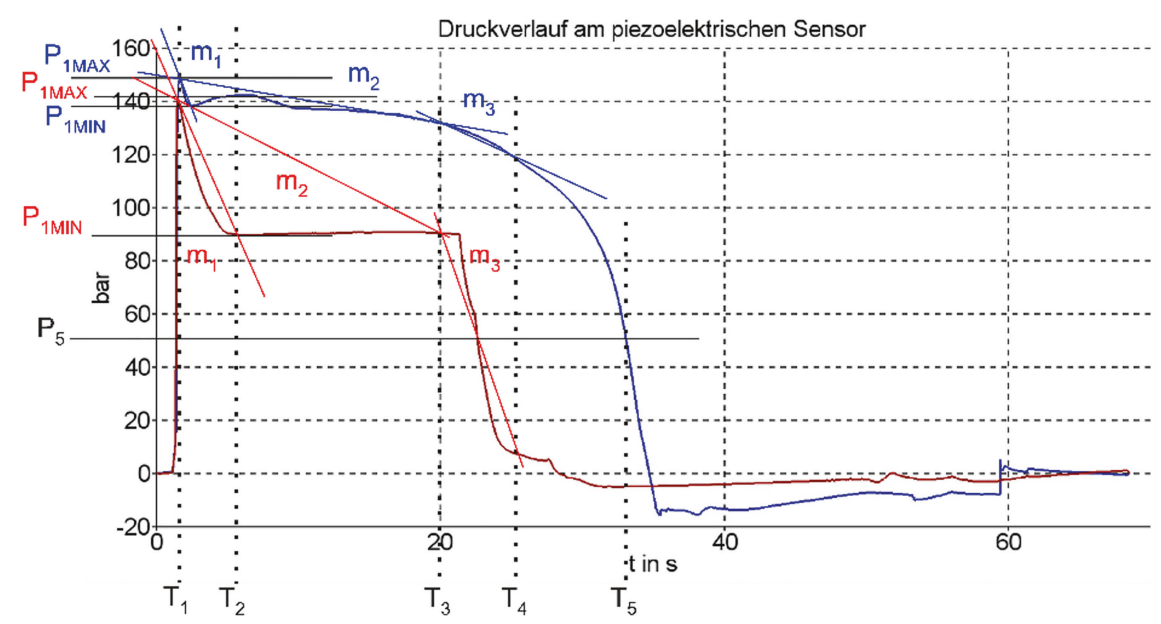

Abb. 4. Projekt Powermould an der Hochschule Schmalkalden. Bewertungskriterien des Fertigungsprozesses. (Bild: Angewandte Kunststofftechnik, Hochschule Schmalkalden)

Durch solche Industrie-4.0-Systeme verbessert jedoch nicht nur der Fertigungsprozess im Sinne von Qualität und Effizienz, es lassen sich über prozessübergreifende Digitalisierungsstrategien gleichermaßen künftige Geschäftsmodelle für den Werkzeug- und Formenbau ableiten: Ähnlich wie im Kfz-Bereich sensibilisieren EDS z. B. für die Instandhaltung von Werkzeugen, wenn dem Nutzer angezeigt werden kann, dass eine Produktionszyklen-Grenze erreicht ist oder das Werkzeug zu großen Kräften ausgesetzt war. Umgekehrt kann der Werkzeugmacher - wenn die notwendigen Parameter vorhanden sind und die Serviceintervalle eingehalten wurden - auch Garantien auf seine Erzeugnisse geben.

\section{Nicht immer alles allein machen! Die kooperative Werkzeugproduktion von Mensch zu Mensch}

Die junge Generation der Werkzeug- und Formenbau-Chefs ist offen und kommunikativ, sie gehen Kooperationen bewusst und gerne ein. Noch vor wenigen Jahren war der Nachbarbetrieb ein gegnerischer Akteur. Die neuen Entscheider begreifen den Kollegen nun jedoch als Bereicherung, als Partner, der mit seinen Ratschlägen weiterhilft, dessen Portfolio das eigene ergänzt oder der mit Kapazitäten helfen kann, um am gemeinsamen Standort Deutschland wirtschaftlich fertigen zu können. Durch diese unternehmerische Philosophie sind in der Branche echte Vertrauensverhältnisse entstanden. Und die Digitalisierung spielt bei den zugehörigen Kommunikationsprozessen eine entscheidende Rolle. Der Austausch von Daten, Einblicke in die Produktion und vor allem der persönlich-menschliche Austausch gelingt mit modernen Medien unkompliziert, direkt und auch über Hunderte Kilometer hinweg.

Notwendige Bedingung für Kooperationen ist jedoch die Flexibilität in der Fertigung. Nur wenn man seine Prozesse digitalisiert und „gläsern“ macht, wenn 
man Informationen zu Programmierungs-, Rüst- oder Bearbeitungszeiten vorliegen hat, kann man auch Arbeitsportionen planbar abgeben bzw. von Kollegen annehmen. Gerade kleine und mittelgroße Unternehmen, die die Werkzeug- und Formenbau-Branche in Deutschland prägen, sind hier im Vorteil. Kleinteiligkeit bedeutet Flexibilität, und Flexibilität bedeutet, dass man schnell handlungsfähig ist. Also keine Angst vor Kleinteiligkeit! Man muss nur intelligent zusammenarbeiten. Wenn es um den Bereich Marketing, Vertrieb und Öffentlichkeitsarbeit geht, tüfteln Werkzeugmacher jedoch manchmal lieber an technologischen Lösungen, als über diese dann zu sprechen. Dabei würde es den vielen „Hidden Champions“ der Branche mit modernen Kommunikations- und Präsentationsmitteln zunehmend leicht gelingen, in den Bereichen Außendarstellung und Vertrieb ihre Kunden und interessierte Unternehmen in der digitalen Welt anzusprechen. ,Tue Gutes und rede darüber“ heißt das Motto.

Für den Marktspiegel Werkzeugbau zeigt sich in den Benchmark-Auswertungen aus dem Jahresabschluss 2019 im Bereich der Digitalisierung und Automatisierung insgesamt ein positiver Trend. So gaben 77,4\% der Unternehmen an, dass sie bereits einzelne moderne Tools zur Digitalisierung einsetzen. Darunter fallen z. B. Augmented-Reality-Lösungen zur Qualitätssicherung, softwaregestütztes und vernetztes Werkzeugmanagement, Virtual-Reality-Lösungen zur Werkzeugabnahme sowie der Einsatz mobiler Endgeräte in der Fertigung. Das Ergebnis zeigt, dass einzelne Tools für die Unternehmer eher greifbar sind und somit leichter integriert werden können als übergreifende Gesamtlösungen (Abb. 5 und 6).

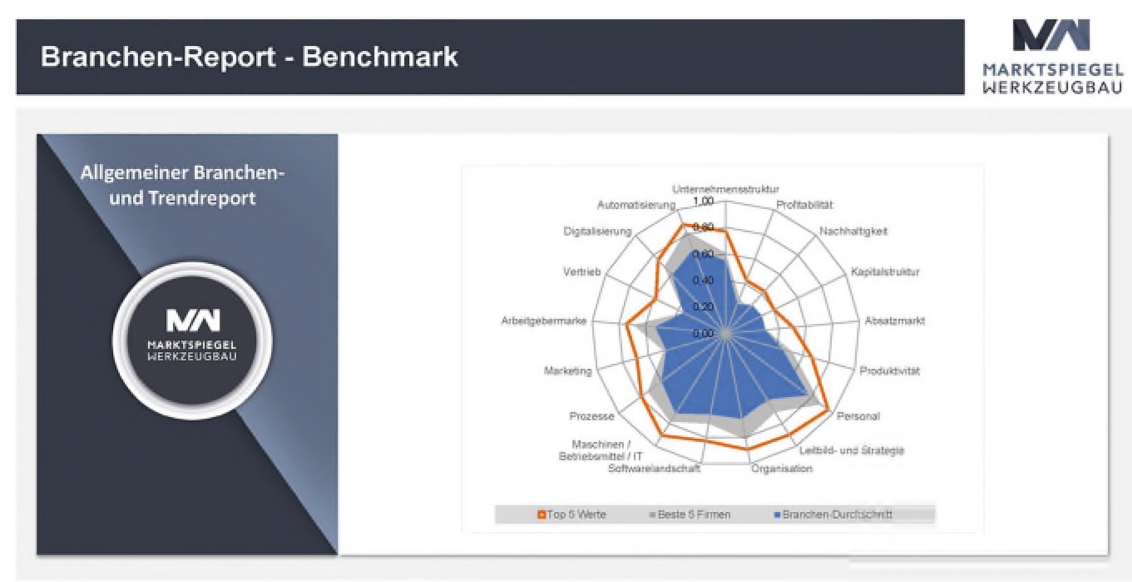

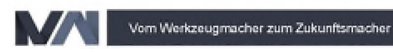

Abb. 5. Marketing und Vertrieb muss zum To-do-Punkt erklärt werden: Laut dem „Marktspiegel Werkzeugbau“ zeigen die Benchmark-Ergebnisse deutlich, dass sich das Marketing- und Vertriebsthema in vielen Unternehmen noch nicht auf der strategischen Agenda befindet. Ähnlich wie auf dem Weg zu einer durchgängigen Digitalisierung gibt es auch hier keine Musterlösung, die jedes Unternehmen adaptieren kann. Wichtig sei, unternehmerischen Mut zu zeigen. Schnell konzipieren, schnell umsetzen und schnell testen, darauf kommt es an. (Bild: Marktspiegel Werkzeugbau, Schwendi) 


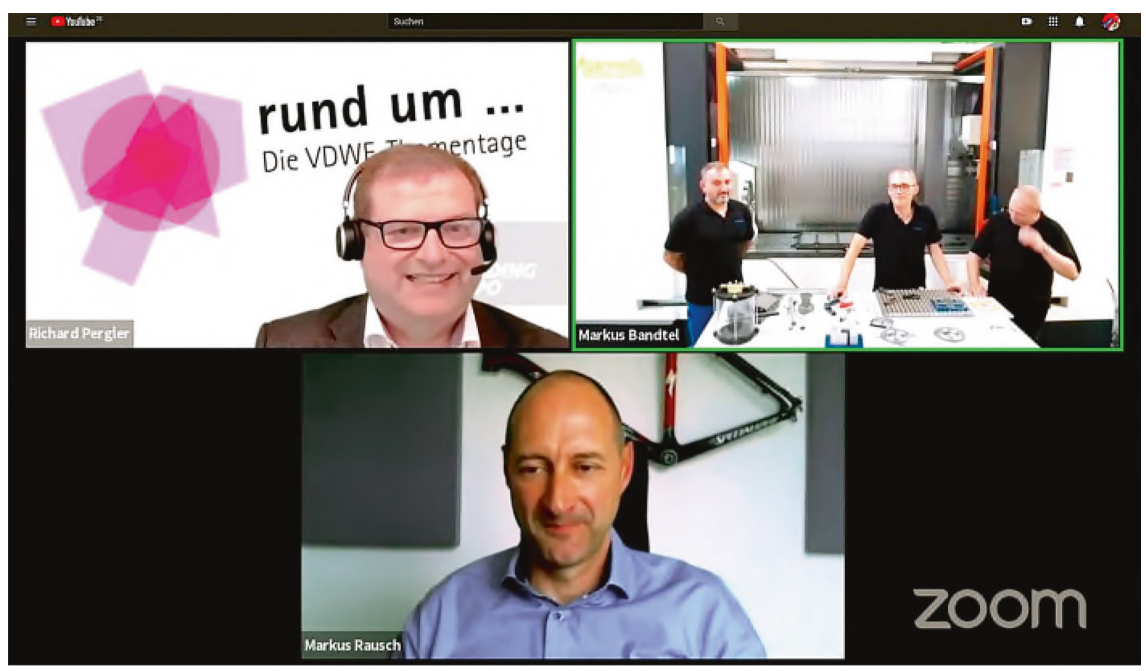

Abb. 6. Mit den Virtuellen Thementagen bietet der VDWF gerade den kleinen und mittelständischen Unternehmen die Möglichkeit, sich ohne große administrative und planerische Hürden ihrer Kundschaft zu präsentieren. Die Reichweite der mit Zoom abgehaltenen Meetings wurde zusätzlich durch Livestreams in YouTube und Facebook erhöht. Ziel ist es, Angebote und Kompetenzen zwischen Branchenpartnern zu vermitteln. (Bild: wortundform GmbH, München)

Bedenken müssen wir dabei aber auch: Die Konkurrenz sitzt mit zunehmender Vernetzung nicht mehr im benachbarten Dorf oder Land, sondern auch auf der anderen Seite der Welt. Deshalb ist es umso wichtiger, zusammenzuarbeiten und gemeinsam für europäische Qualität zu stehen. Die Werkzeugmacher hierzulande agieren bereits international. In Richtung Nord- und Südamerika, Brasilien, Mexiko, Kanada, aber auch in Indien wird sich jedoch bald der Markt erweitern. Doch nur die großen Betriebe können langfristig international erfolgreich agieren - um umgekehrt, Betriebe mit 50 oder mehr Mitarbeitern müssen auch international operieren, um überlebensfähig zu sein, um die eigenen Kapazitäten auszuschöpfen. In einem globalisierten Wettbewerb darf man nicht innehalten. Um effizient zu sein und damit auch nachhaltig wettbewerbsfähig, gilt es nicht nur Prozessabläufe in der Produktion, sondern gleichermaßen auch in der Verwaltung zu optimieren. Ein Lösungsansatz für die kleineren Unternehmen kann hier die kooperative Fertigung sein. Man kann also unternehmensübergreifende Allianzen eingehen, um gemeinsam einen größeren Auftrag zu stemmen, anstatt ihn Dritten zu überlassen. Gekoppelt mit Werkzeugbau 4.0 ermöglicht das ganz neue Geschäftsmodelle für den Werkzeug- und Formenbau - immer nach dem Motto ,Think global, act local!“‘. Das ist nicht nur eine Zukunftsvision, sondern die Zukunft (Abb. 7). 


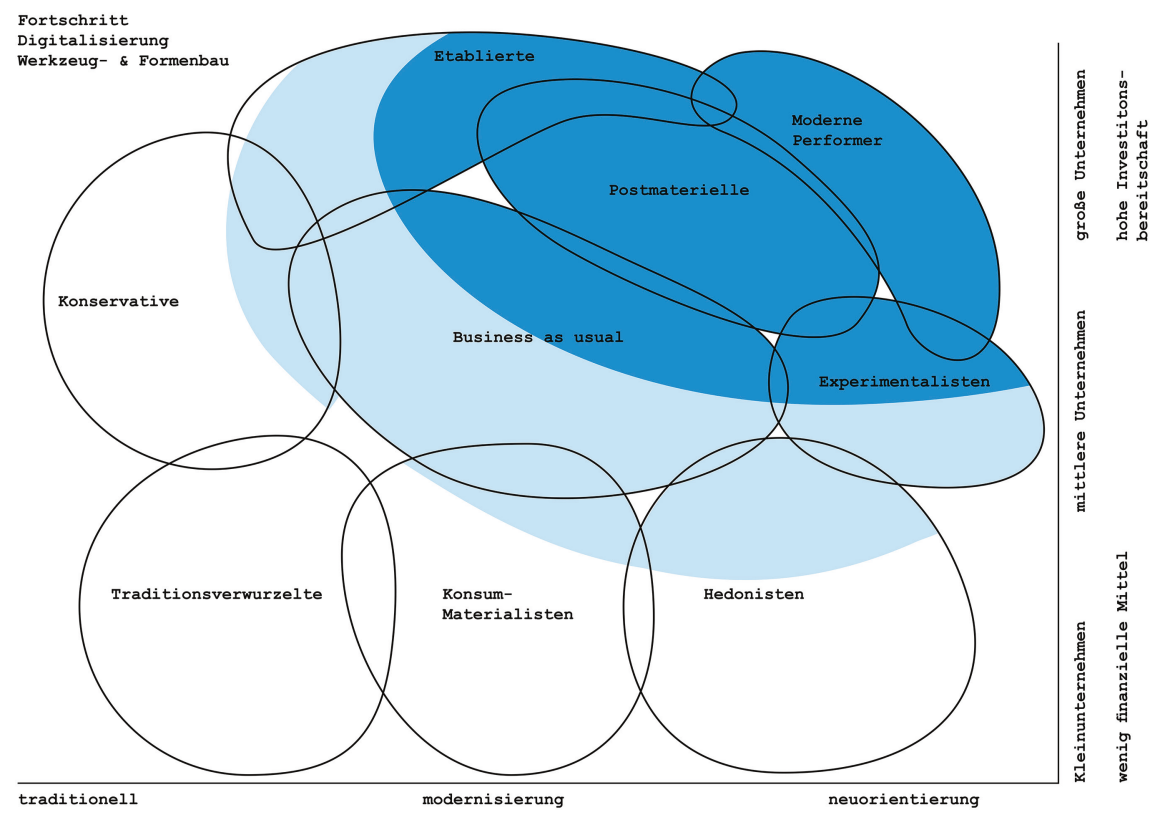

Abb. 7. Fortschritt der Digitalisierung im Werkzeug- und Formenbau. (Bild: wortundform $\mathrm{GmbH}$, München)

\section{Ohne Bildung, ohne Forschung kein Erfolg}

Nur die Ausbildung macht uns hier in Deutschland überlebensfähig! Die Erstausbildung findet auf höchstem Niveau statt aber sie befähigt uns nicht dazu, bis zum Ruhestand unsere Aufgabe - in diesem volatilen Markt, mit ständig neuen Technologieentwicklungen - adäquat zu erfüllen. Früher gab es Werker, die arbeiteten bis zum Ruhestand an derselben Maschine. Das wird es nicht mehr geben. Um das lebenslange Lernen kommen wir nicht mehr herum. Daher haben sich gerade im Werkzeugund Formenbau verschiedene Weiterbildungs-Studiengänge etabliert (Abb. 8). Denn nur die Qualifizierung - nicht nur der Anlagen, Werkstoffe und Prozesse, auch der Menschen - macht Standorte mit hohem Lohnniveau überlebensfähig! 


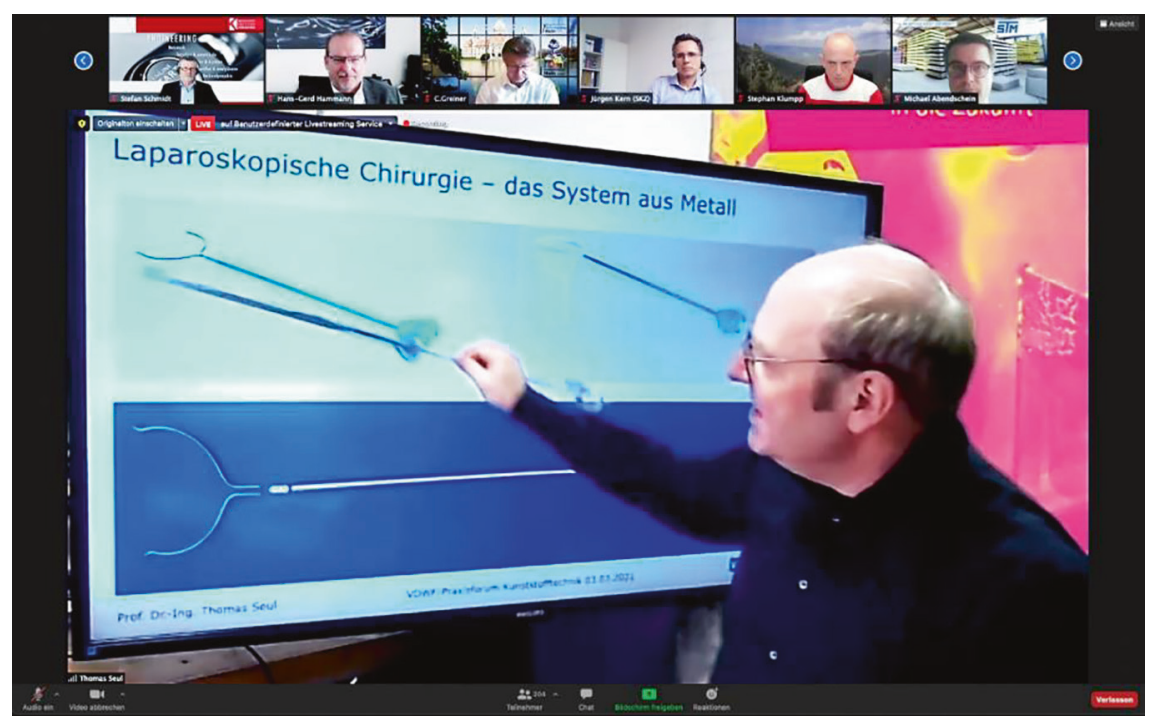

Abb. 8. Reichweitenerhöhung in der Werkzeug- und Formenbau-Branche: VDWF-Präsident Prof. Thomas Seul, auch Vizepräsident für Forschung und Transfer an der Hochschule Schmalkalden, erläutert bei dem rein digital veranstalteten „Praxisforum Kunststofftechnik“ die Vorteile eines medizinischen Bergebeutels aus Kunststoff. (Bild: wortundform GmbH, München)

Als Musterbeispiel für den Umgang mit Digitalisierung und Standardisierung kann hier die Additive Fertigung dienen: Bei diesem relativ jungen Fertigungsverfahren, das ein digitale Planung und Fertigung der Bauteile zwingend bedingt, wurde lange Zeit nicht einmal eine Erstausbildung angeboten. Erst als den viele Autodidakten, die sich dem Thema widmeten, eine generelle Basis von Standards und Richtlinien geboten wurde, gelang es in Bezug auf Werkstoff- und Verfahrenseigenschaften, in Bezug auf Engineering, aber auch in Bezug auf die Werkzeugkonstruktion einen zielführenden Austausch stattfinden zu lassen. Bei Qualitätssicherung, Technologiebewertung und -handhabung oder einfach auch beim Einstellen neuer Mitarbeiter sind vereinheitlichte Daten wesentliche Basis. 
„3D“ hat uns gezeigt, dass es sich lohnt, generell in der Konstruktion von Produkten umzudenken. Vereinbarte Standards bei Werkstoffen, Normalien, Schnittstellen und Prozessabläufen sind die Basis, damit die Ergebnisse aus der Forschung effektiv und auch effizient in die Werkzeug- und Formenbaufertigung transferiert werden können. Gerade auch bei kollaborativen Projekten, bei denen verschiedene Forschungseinrichtungen und Unternehmen zusammenarbeiten. Auf der anderen Seite helfen Digitalisierung und Standardisierung auch, wenn es darum geht, Forschungsthemen zu identifizieren. Meistens sind es die wissenschaftlichen Einrichtungen, die auf die Unternehmen zugehen, doch eigentlich sollte der Weg umgekehrt sein und die Industrie sollte mitgestalten und die Unternehmen der Forschung sagen, welche Themen in den nächsten fünf, zehn oder fünfzehn Jahren ins Programm gehören. Sie müsste die weißen Felder identifizieren und gewichten, damit Forschungsthemen priorisiert werden können. Dies funktioniert erst ideal, wenn auf beiden Seiten dieselben Standards gelten und auf dieselbe Datenbasis verwiesen werden kann (Abb. 9).

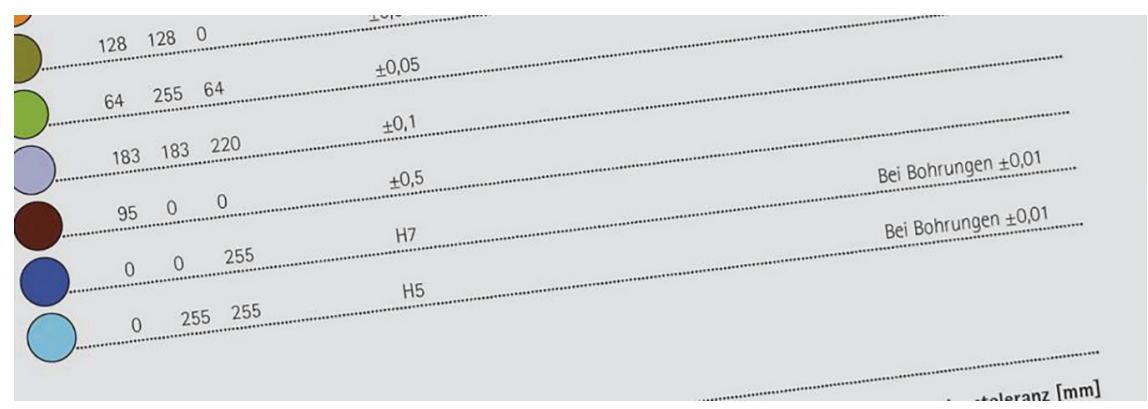

Abb. 9. VDWF-Richtlinie „Farbtabelle zur CAD-gestützten Übermittlung von Toleranzen im Werkzeug- und Formenbau“: Werkzeugmacher färben Flächen von CAD-Modellen ein, um die erforderliche Fertigungsgenauigkeit in der Konstruktion festzulegen. So lassen sich die Prozesse der Unikatfertigung rationalisieren. Allerdings wurde der Effekt dieses sinnvollen Vorgehens in den letzten Jahren dadurch eingeschränkt, dass zahlreiche Betriebe eigene Farbtabellen erstellt haben und eine Vielzahl unterschiedlicher Systeme kursierten. Dadurch kam es im Produktionsalltag immer wieder zu „Gewohnheitsfehlern“, wenn Werkzeug- und Formenbau-Unternehmen beispielsweise einzelne Teilaufgaben, wie z. B. Fräs-, Bohr- oder Erodierarbeiten, an externe Dienstleister vergaben. Da aber ein weiterer Anstieg des Terminund Kostendrucks zu erwarten ist, wird die Notwendigkeit vernetzten Arbeitens zunehmen. Hier soll die vom VDWF erarbeitete Farbtabelle Übermittlungsfehler verhindern. In enger Kooperation erarbeitete eine Expertenrunde aus der Branche die vereinheitlichende Richtlinie zur CAD-gestützten Übermittlung von Toleranzen im Werkzeug- und Formenbau per Farbcodierung. (Bild: wortundform GmbH, München)

\section{Mit Effizienz, ohne Effizienz}

Verändert sich die Arbeit, verändert sich auch die Atmosphäre bei der Arbeit. Die heutigen effizienzgetriebenen Prozesse lassen Beschaulichkeit nicht mehr zu. Wenn früher etwas fertig werden musste und alle auch samstags im Betrieb waren, 
frühstückte man gemeinsam und tauschte sich aus. So lernte man die Menschen in den Kollegen kennen, ihre Meinungen, ihre Gefühle - was für das Selbstverständnis und die Identifikation der Werkzeugmacher mit ihrem Berufsbild und mit ihrem Arbeitgeber wesentlich war. Werden nun alle Abläufe im Unternehmen auf Effizienz getrimmt, besteht die Gefahr, dass an anderer Stelle etwas verloren geht. Es gilt also, sich mit Bedacht zu verändern und das wertvollste Gut in unserer Branche - den Menschen - stets in den Mittelpunkt der Digitalisierungsstrategien zu setzen.

$\mathrm{Zu}$ guter Letzt gilt es, auch als Werkzeugmacher keine Angst zu haben: Mit digitalisierten und automatisierten Prozessen wird niemand arbeitslos, vielmehr gewinnt man nun die Zeit, um sich aufs Wesentliche konzentrieren zu können - nämlich auf die Arbeiten, die den Menschen mit seiner Erfahrung benötigen und nicht von Robotern erledigt werden können. Und: Digitalisierung ist kein Allheilmittel, Software ist nicht intelligent. Intelligent wird ein System erst dadurch, dass wir Menschen mit der Software umgehen. Das ist übrigens in allen Bereichen so. Ein Architekt konstruiert per CAD ein Haus, doch der Spirit des Entwurfs, die Raumatmosphäre, das Zusammenspiel der Proportionen, das macht der gestaltende Akteur und nicht die Software. CAD-Systeme und -Programme sind die Werkzeuge, um dem zu helfen, der kreative Gedanken umsetzen möchte!

Die Automatisierung mit digitalisierten Prozessen als Basis wird also eintönige, wiederkehrende Arbeitsschritte übernehmen und ersetzen und neue Freiräume schaffen. Der Mensch wird mit seinem erarbeiteten Wissen aus früheren Projekten dabei immer Teil des Ganzen bleiben. Denn ohne den Werkzeugmacher geht nichts. Weder in der Vergangenheit noch in der Zukunft.

Open Access Dieses Kapitel wird unter der Creative Commons Namensnennung 4.0 International Lizenz (http://creativecommons.org/licenses/by/4.0/deed.de) veröffentlicht, welche die Nutzung, Vervielfältigung, Bearbeitung, Verbreitung und Wiedergabe in jeglichem Medium und Format erlaubt, sofern Sie den/die ursprünglichen Autor(en) und die Quelle ordnungsgemäß nennen, einen Link zur Creative Commons Lizenz beifügen und angeben, ob Änderungen vorgenommen wurden.

Die in diesem Kapitel enthaltenen Bilder und sonstiges Drittmaterial unterliegen ebenfalls der genannten Creative Commons Lizenz, sofern sich aus der Abbildungslegende nichts anderes ergibt. Sofern das betreffende Material nicht unter der genannten Creative Commons Lizenz steht und die betreffende Handlung nicht nach gesetzlichen Vorschriften erlaubt ist, ist für die oben aufgeführten Weiterverwendungen des Materials die Einwilligung des jeweiligen Rechteinhabers einzuholen.

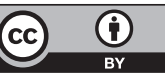

